\title{
A novel CMOS sensor for measuring thermal diffusivity of liquids
}

\author{
Yi-Ting Cheng, Chih-Wei Chang, Ya-Rui Chung, Jui-Hung Chien, \\ Jin-Sun Kuo, Wei-Ting Chen, Ping-Hei Chen* \\ Department of Mechanical Engineering, National Taiwan University, No. 1, Sec. 4, Roosevelt Rd., Taipei, Taiwan 10617, ROC \\ Received 19 March 2006; received in revised form 5 July 2006; accepted 31 August 2006 \\ Available online 6 October 2006
}

\begin{abstract}
This study aims at designing and developing a CMOS sensor chip for measuring thermal diffusivity of liquids. The CMOS sensor chip is realized by VIS $0.5 \mu \mathrm{m} 2 \mathrm{P} 3 \mathrm{M}$ CMOS process with maskless post-CMOS micromachinings. The procedure used to measure thermal diffusivity of liquids is to replace conventional methods that require a great deal of specimen and take a long measuring time. The CMOS chip consists of a thin film of polysilicon covering a cavity of the substrate. The thin film is equipped with a rectangular centered heater and four temperature sensors that are located at different locations from the heater. Once a known voltage is applied to the heater, the thermal diffusivity of the liquid drop that spreads over the heater and the temperature sensors can be determined from the measured temperature responses of the temperature sensors. Different kinds of tested liquids result in different temperature variations. The temperature variation corresponds to the resistance variation of temperature sensors. Three kinds of liquids were measured in this study. The conclusions drawn from the experimental results are as follows: high thermal diffusivity of a liquid causes large heat flux in vertical direction and small heat flux in horizontal direction, thus there is small resistance variation of the temperature sensor. In addition, liquid with high thermal diffusivity gives the experiment system a large time constant. Thermal diffusivities of other liquids can be measured based on a relation between time constant and thermal diffusivity derived from the tested liquids.

(C) 2006 Elsevier B.V. All rights reserved.
\end{abstract}

Keywords: CMOS sensor; Thermal diffusivity; Post-CMOS micromachining

\section{Introduction}

Over the past several decades, numerous methods have been proposed on the measurement of the thermal diffusivity of liquids under different ranges of temperature and pressure. These methods can be classified into two types [1]; namely, steadystate and unsteady-state methods. The steady-state methods are well developed, but are expensive in terms of equipment and are difficult to operate. Among various unsteady-state methods presented so far, a transient hot-wire method [2] becomes one of the commonly used techniques for the measurement of thermal diffusivity of liquids. Tomimura et al. [3] developed a transient short-hot-wire method which has been applied to the refrigerant HCFC-22, alternative refrigerants HFC-32, HFC-125, HFC134a and refrigerant mixtures HFC-32/125, HFC-32/125/134a.

\footnotetext{
* Corresponding author. Tel.: +886 223670781; fax: +886223670781.

E-mail address: phchen@ntu.edu.tw (P.-H. Chen).
}

Nagasaka and Nagashima [4] developed an apparatus for measuring the thermal diffusivity of electrically conducting liquids, based on transient hot-wire method. They overcame the problem of electrically conducting liquids by using a metallic wire with a thin electrically insulation layer instead of just the bare metallic wire. The transient hot-wire provides a rapid, absolute means for measuring the thermal diffusivity of liquids.

Another common transient method for measuring thermal diffusivity is a transient hot-strip method developed by Gustafsson et al. [5]. The transient hot-strip method is similar to the transient hot-wire method. A transient plane source (TPS) element, which is both a heat source and temperature sensor, is used in the same way as a thin wire is used in the transient hotwire method. TPS element is made of thin metal foil and its conducting pattern is a double spiral with some approximation resembling a hot disk. For the transient hot-wire method, a thin bare wire has been utilized.

Without coating an insulation thin layer, only the thermal diffusivity of insulating materials can be measured. In contrast, 


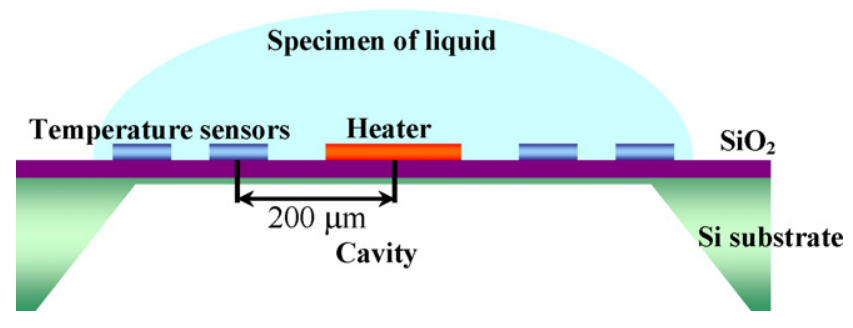

Fig. 1. Cross-sectional view of a novel CMOS sensor chip for measuring the thermal diffusivity of liquids.

the thermal diffusivity of either insulating liquids or electrically conducting liquids can be measured by the transient hot-strip method because both sides of TPS elements are covered with a thin insulating layer.

Though the transient hot-wire method and the transient hotstrip method are common methods used to measure the thermal diffusivity of liquids, they usually need large volumes of liquid for measuring, and the apparatus and data reduction procedures are complex for routine use. Some of the liquids are very expensive and not easy to obtain, such as nano-fluids, magnetic nano-fluids and medical drugs. It is a challenge to fabricate a miniature device which is equipped with signal processing circuits and requires only little specimen volume for measuring thermal diffusivity of liquids. The complementary metal oxide semiconductor (CMOS) fabrication is a possible way to fit the criteria mentioned above. In recent years, papers about thermal sensors using CMOS fabrication process have been published [6-9], but the measurement of thermal properties of liquids is not discussed. The aim of this study is to fabricate a novel CMOS-MEMS sensor for measuring thermal diffusivity of liquids through a commercially available $0.5 \mu \mathrm{m}$ mixed signal 2P3M CMOS process provided by Vanguard International Semiconductor Corporation (VIS).

\section{Design principle}

Fig. 1 illustrates the cross-section view of the main system of the CMOS sensor chip and Fig. 2 shows the whole chip layout. This measuring system mainly includes a heater at the center, temperature sensors, specimen of liquid drop and a cavity for thermal insulation. The heater and temperature sensors are made of polysilicon due to its high resistance and the large resistance variation with temperature changes.

Before the heater is heated up, the temperature of the whole system is the same as the ambient temperature. Once a heat flux is suddenly generated by the heater, it will cause temperature variations of the sensor. Different temperature variations would be observed due to different kinds of specimens. The temperature response of the sensor will be used to determine the thermal diffusivity of liquids if most heat is transported into the liquid from the heater.

\section{Post-CMOS micromachining}

In order to fabricate the miniature CMOS sensor, the chips are fabricated using $0.5 \mu \mathrm{m} 2 \mathrm{P} 3 \mathrm{M}$ CMOS process developed by

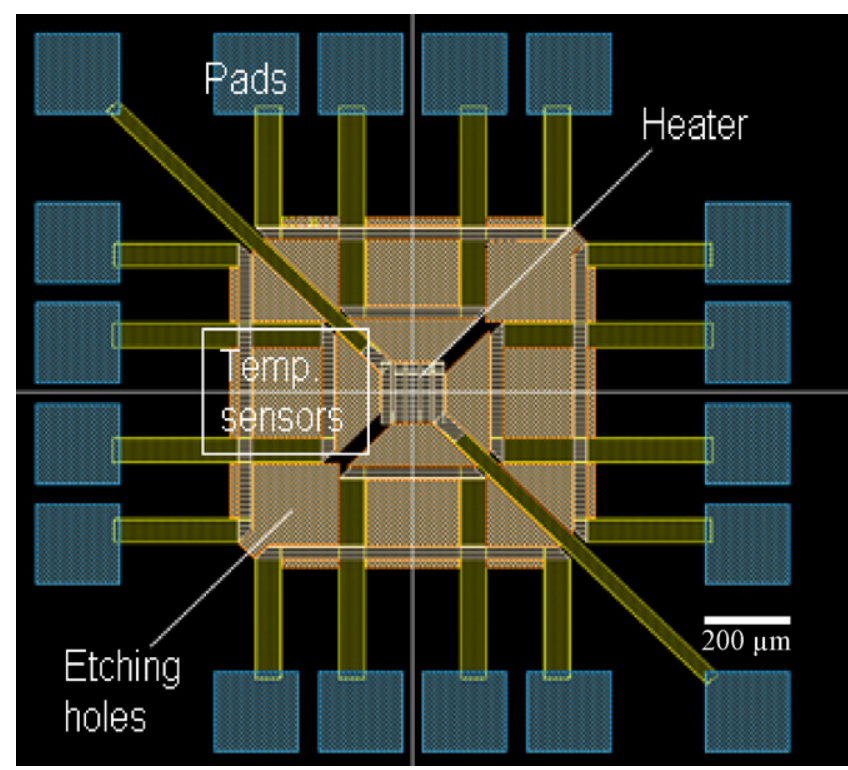

Fig. 2. Layout of the CMOS sensor chip.

VIS. After CMOS fabrication process, post-CMOS micromachinings will be required to complete the microstructures. The aim of the micromachining here is to release the microstructures used as heater and temperature sensors in this design. Post-CMOS micromachining processes are as follows: wet etching for removing metal layers; dry etching for removing silicon dioxide; wet etching for removing silicon substrate; dry etching for opening pads; wire bonding. The whole etching processes are realized by the concept of maskless etching. As implied in the name, additional photoresists and masks are not necessary in the post-CMOS processes. It greatly increases the possibility of success in the fulfillment of microstructures. Fig. 3 shows the sketch of the flow chart of post-CMOS micromachining processes. Fig. 3(a) is the tapped-out chip after fabrication by VIS standard CMOS process. Fig. 3(b) illustrates the use of isotropic wet etching to remove the metal layers with a traditional Piranha solution at $80^{\circ} \mathrm{C}$ for $30 \mathrm{~min}$. The etchant is a $3: 1$ mixture of concentrated sulfuric acid $\left(\mathrm{H}_{2} \mathrm{SO}_{4}\right)$ and hydrogen peroxide $\left(\mathrm{H}_{2} \mathrm{O}_{2}\right)$. The isotropic wet etching solution flows and etches along the etching holes that are originally filled with metal structures without passivation layers on them. Fig. 3(c) shows the sketch of anisotropic dry etching-reaction ion etching (RIE) to remove the thin $\mathrm{SiO}_{2}$ layer on the silicon substrate. The RIE process is employed by using gas flows of $\mathrm{CF}_{4}$ at a flow rate of $50 \mathrm{sccm}$ under a vacuum pressure of $7.8 \mathrm{~Pa}$ with an RF power of $70 \mathrm{~W}$ for $8 \mathrm{~min}$. Fig. 3(d) shows the use of anisotropic wet etching to remove silicon under the heater and temperature sensors. Some parts of the silicon substrate are exposed to the air after $\mathrm{SiO}_{2}$ layer is removed by the RIE process. The common anisotropic wet etching solution for silicon is TMAH, which is also compatible with CMOS process [10-12]. In this study, we used $8 \mathrm{wt} . \%$ TMAH solution at $80^{\circ} \mathrm{C}$ for $4 \mathrm{~h}$ to remove silicon. Fig. 3(e) shows the use of anisotropic dry etching-reaction ion etching (RIE) to remove passivation and $\mathrm{SiO}_{2}$ layer again. The RIE process is employed by using gas flow of $\mathrm{CF}_{4}$ at a flow rate of $50 \mathrm{sccm}$ under a vacuum pressure of $7.8 \mathrm{~Pa}$ with 
(a)

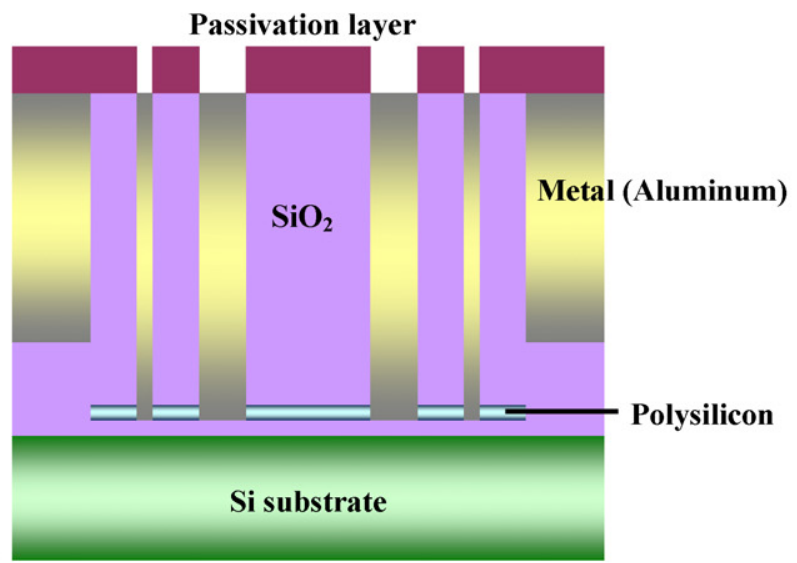

(b)

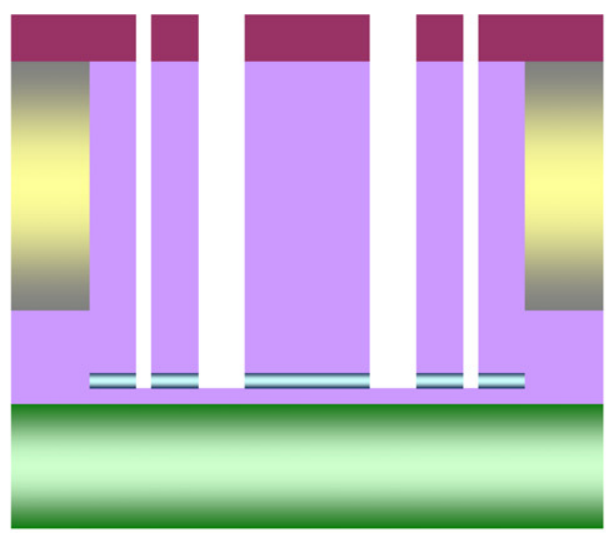

(c)

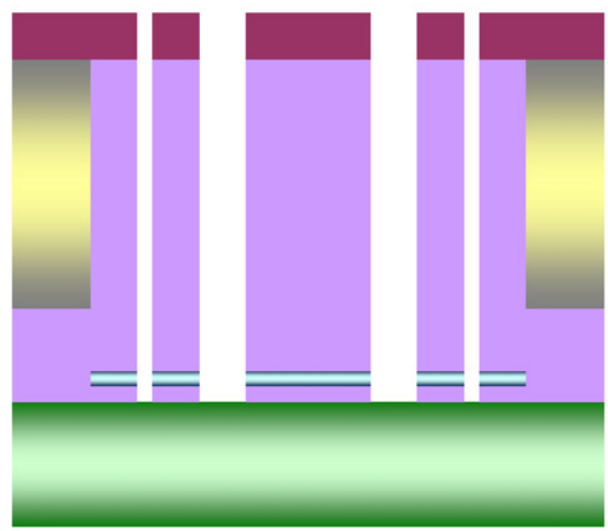

(d)

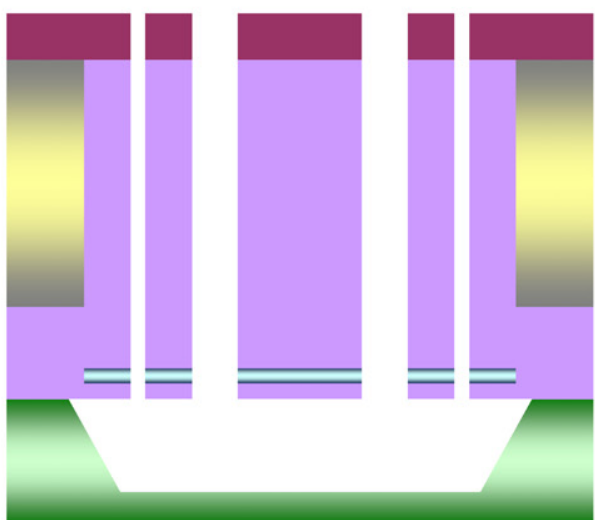

(e)

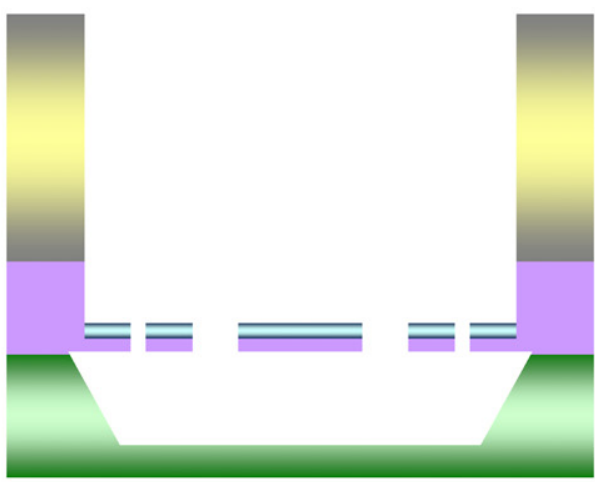

(f)

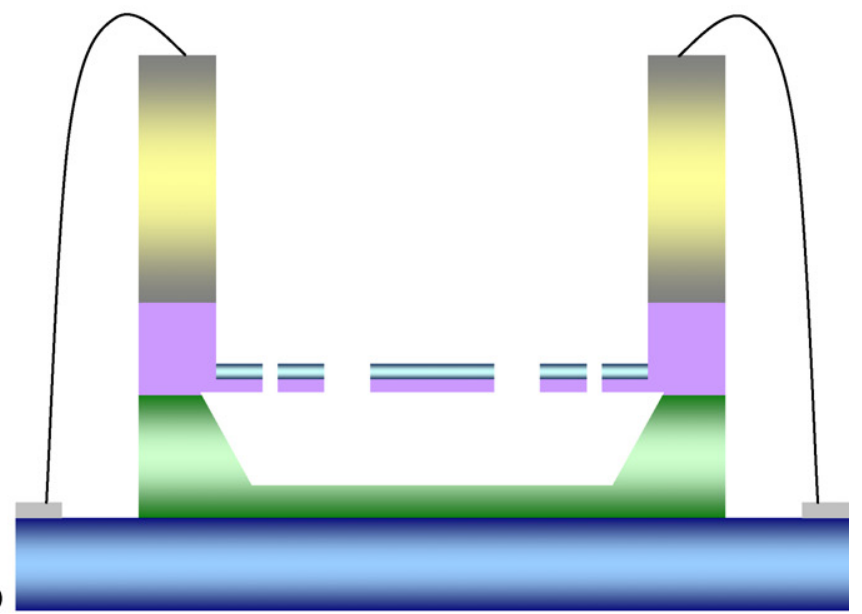

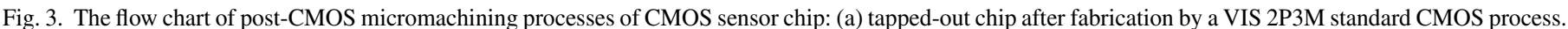

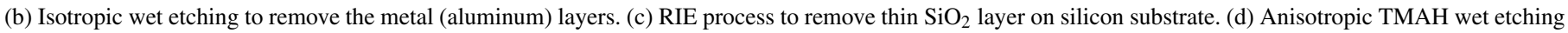

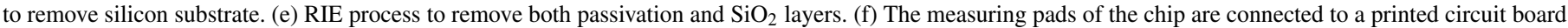
by a wire bonding technique.

an RF power of $70 \mathrm{~W}$ for $8 \mathrm{~min}$. Due to etching selectivity of RIE between different materials, the microstructures are formed without any additional masks and photoresists. Fig. 3(f) shows that the measuring pads of the chip are connected to printed circuit board (PCB) by wire bonding technique for verification of the function of the chip. Fig. 4 is the SEM micrograph of the CMOS sensor chip after the post-CMOS micromachining process.

\section{Experiment procedures}

\subsection{Procedures of resistance and temperature calibration}

After microstructures of the CMOS sensor chip are fulfilled by post-CMOS micromachinings, a calibration procedure is needed to identify the relation between the resistance and the temperature of temperature sensors (polysilicon). In the 


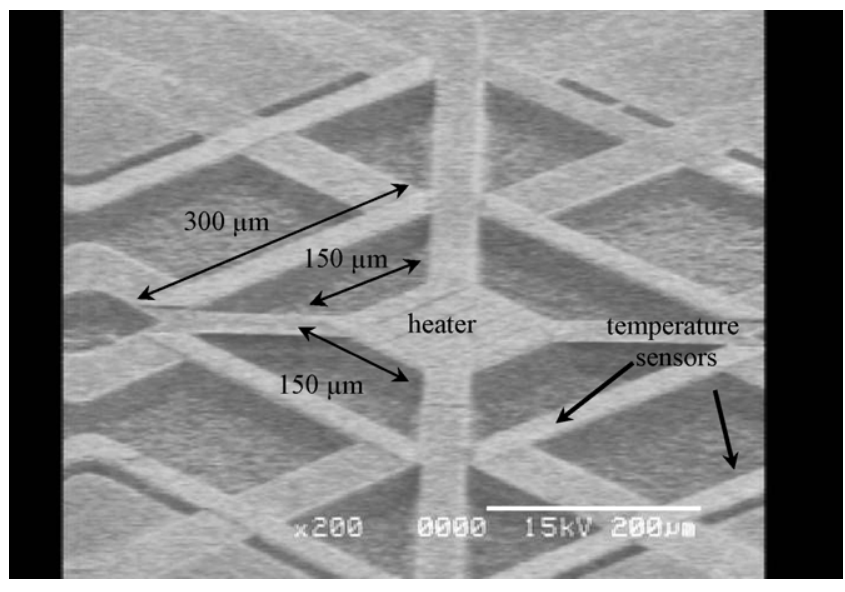

Fig. 4. SEM micrograph of the CMOS sensor chip after post-CMOS micromachining processes.

calibration procedure, the CMOS sensor chip is bedded in a constant-temperature container which allows the adjustment of temperature. A thermocouple is also used to confirm the realtime temperature in the constant-temperature container. The resistance of the sensor is measured by a digital multimeter. Fig. 5 shows the calibration result of temperature versus resistance of temperature sensor. It is clearly seen that the resistance always decreases linearly with increasing temperature since the material used for sensors is undoped polysilicon. Fig. 5 also illustrates the fitting curve of calibration, and a nearly linear relation between resistance and temperature of the sensor can be obtained and expressed as follows:

$R=29.0499-0.1119 T$

where $R$ is the resistance of the sensor $(\mathrm{k} \Omega)$ and $T$ is the temperature in Kelvin (K).

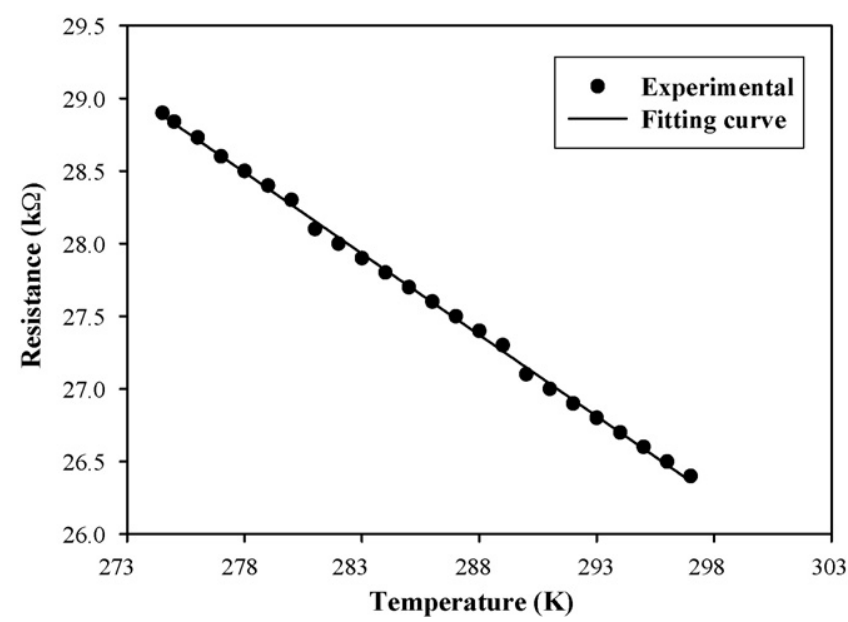

Fig. 5. The calibration of resistance and temperature of the polysilicon temperature sensor.

\subsection{Procedures of measuring thermal diffusivity of tested liquids}

Due to the low boiling point of some of the tested liquids, the ambient temperature of the measuring system should be as low as possible to avoid evaporation. For this reason, the CMOS sensor chip with PCB is set in the constant-temperature container controlled at $274.5 \mathrm{~K}$. An AC power supply and a data logger are also connected to the chip. The required volume of each specimen is $3 \mu \mathrm{L}$ to form a liquid drop spreading over the thin film consisting of a heater and several temperature sensors. Each specimen is dripped on the chip by a pipette to control the liquid volume precisely. In addition, a thin Teflon film is coated on the circuit and metallization structures to protect the tested liquids from electric current. A proper voltage is applied across the heater to generate $0.01 \mathrm{~W}$ power once the temperature of CMOS sensor chip and tested specimen reach the ambient temperature

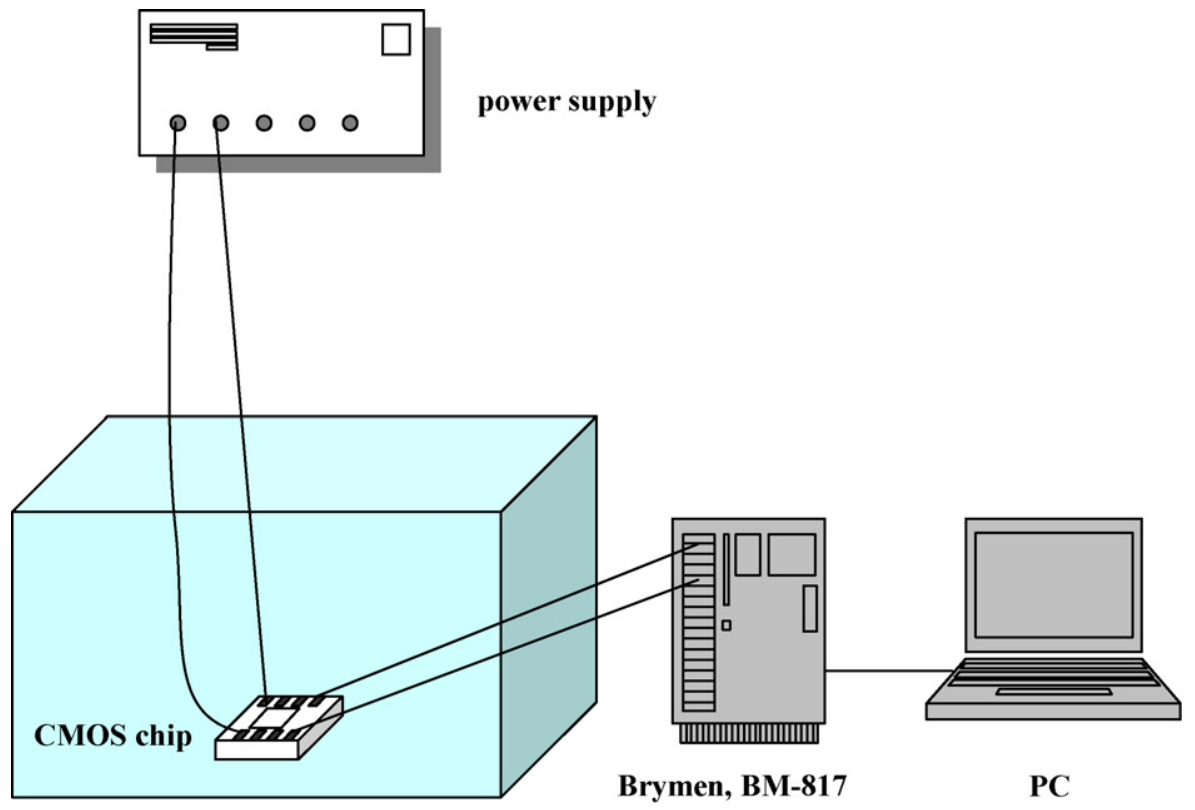

Fig. 6. A schematic view of the experimental setup. 


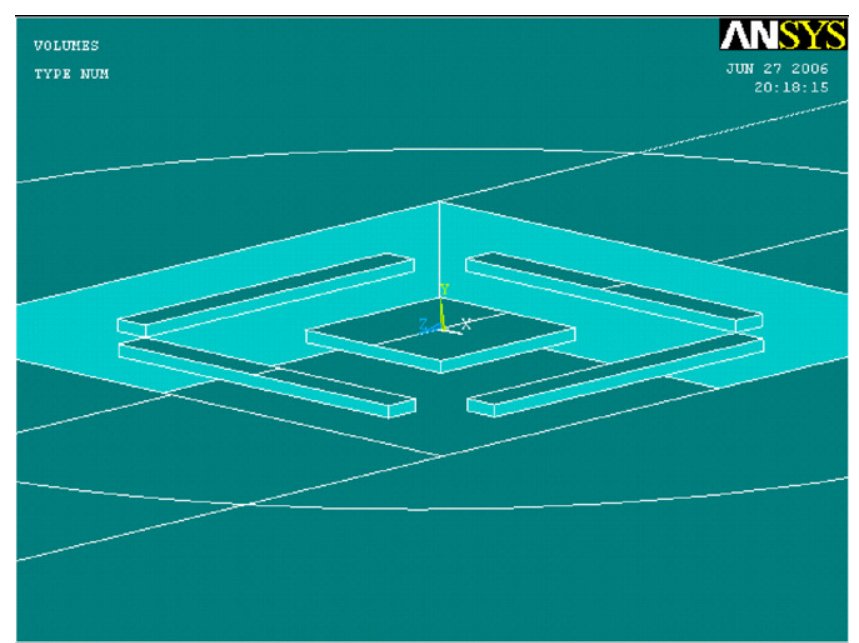

Fig. 7. ANSYS solid model of the whole CMOS sensor chip.

(274.5 K). The heat flux will cause a temperature variation of the sensor. The real-time temperature can be calculated from the resistance of the temperature sensors. Resistance variation of sensors is recorded by a data logger. Fig. 6 illustrates a schematic view of the experimental setup.

\section{ANSYS simulation}

ANSYS software simulation of the novel microdevice for measuring the thermal diffusivity of liquids is described in this section. The solid model consists of liquids, aluminum, silicon, silicon dioxide and polysilicon thin film. The whole solid model is shown in Fig. 7. The element type of Solid 90 is applied to silicon substrate and liquid. The element type of Shell 57 is applied to aluminum, silicon dioxide and polysilicon thin film. The dimension of the silicon substrate block is $2000 \mu \mathrm{m}$ in length and width and $500 \mu \mathrm{m}$ in thickness. And the thickness of polysilicon is $2700 \AA$. The polysilicon thin film is used as heater and temperature sensor. The dimension of heater, which is at the center of the microdevice, is $150 \mu \mathrm{m}$ in length and width. The temperature sensors are $30 \mu \mathrm{m}$ in width and at a distance of $200 \mu \mathrm{m}$ away from the center.

The initial condition is set as $274.5 \mathrm{~K}$, and the boundary conditions are applied as follows: heat generation $(0.01 \mathrm{~W})$ through the heater is $1.65 \times 10^{12} \mathrm{~W} / \mathrm{m}^{3}$ and a convection boundary condition is applied along the intersection surface between polysilicon and air.

Fig. 8 shows the temperature distribution of the whole CMOS sensor chip and the used parameters of tested liquid is toluene. In this case, the temperature variation of temperature sensor is $15.03{ }^{\circ} \mathrm{C}$. Fig. 9 illustrates the simulation results of temperature variation of the sensor with different tested liquids during processing time.

\section{Results and discussions}

When heat flux is generated from the heater, the heat will pass through the liquid drop and the temperature of the sensor will change simultaneously. The temperature variation can be

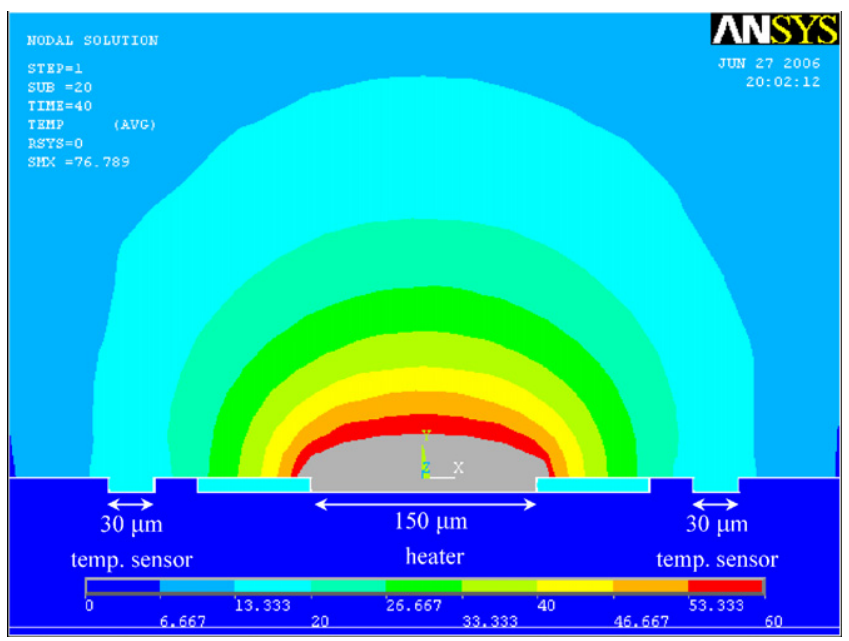

Fig. 8. ANSYS simulation result of the whole CMOS sensor chip temperature distribution and the used parameters of tested liquid is toluene.

obtained from the resistance change detected by the sensor in this study. Fig. 10 illustrates the resistance variation of the sensor with different tested liquids during processing time. The experimental results could be fitted by Eqs. (2)-(4) as the impulse response of an RC lowpass filter to a unit step signal.

Toluene $\frac{\Delta R}{R_{\text {in }}}=4.328\left(1-\mathrm{e}^{-t / \tau}\right), \quad t=3.11$

Ethanol $\frac{\Delta R}{R_{\text {in }}}=4.1911\left(1-\mathrm{e}^{-t / \tau}\right), \quad t=3.99$

Glycerin $\frac{\Delta R}{R_{\text {in }}}=2.93\left(1-\mathrm{e}^{-t / \tau}\right), \quad t=6.66$

Experimental results and fitting curves are denoted by a normalized $R / R_{\text {in }}$ factor, where $R_{\text {in }}$ is the initial resistance. The simulation results (Fig. 9) and experimental results (Fig. 10) show the same trend of temperature response curves of temperature sensor.

The time response of the experimental results is characterized by a time constant $(\tau)$ in the equation of the fitting curve since different system behaviors could be illustrated by individual time constants. It can be observed that a liquid with higher thermal diffusivity corresponds to smaller resistance and temperature variations of the temperature sensor. Table 1 shows the comparison between the resistance and temperature variation among these liquids.

A liquid with higher thermal diffusivity will have a larger characteristic time constant. This phenomenon can be explained as follows: when a liquid is heated by the heater, the heat flux

Table 1

The comparison of resistance and temperature variation among three tested liquids at steady-state

\begin{tabular}{lclr}
\hline & $\alpha \times 10^{8}\left(\mathrm{~m}^{2} / \mathrm{s}\right)$ & $\Delta R(\mathrm{k} \Omega)$ & $\Delta T(\mathrm{~K})$ \\
\hline Toluene & 8.661 & 1.26 & 11.51 \\
Ethanol & 9.083 & 1.20 & 10.91 \\
Glycerin & 10.088 & 0.87 & 7.89 \\
\hline
\end{tabular}



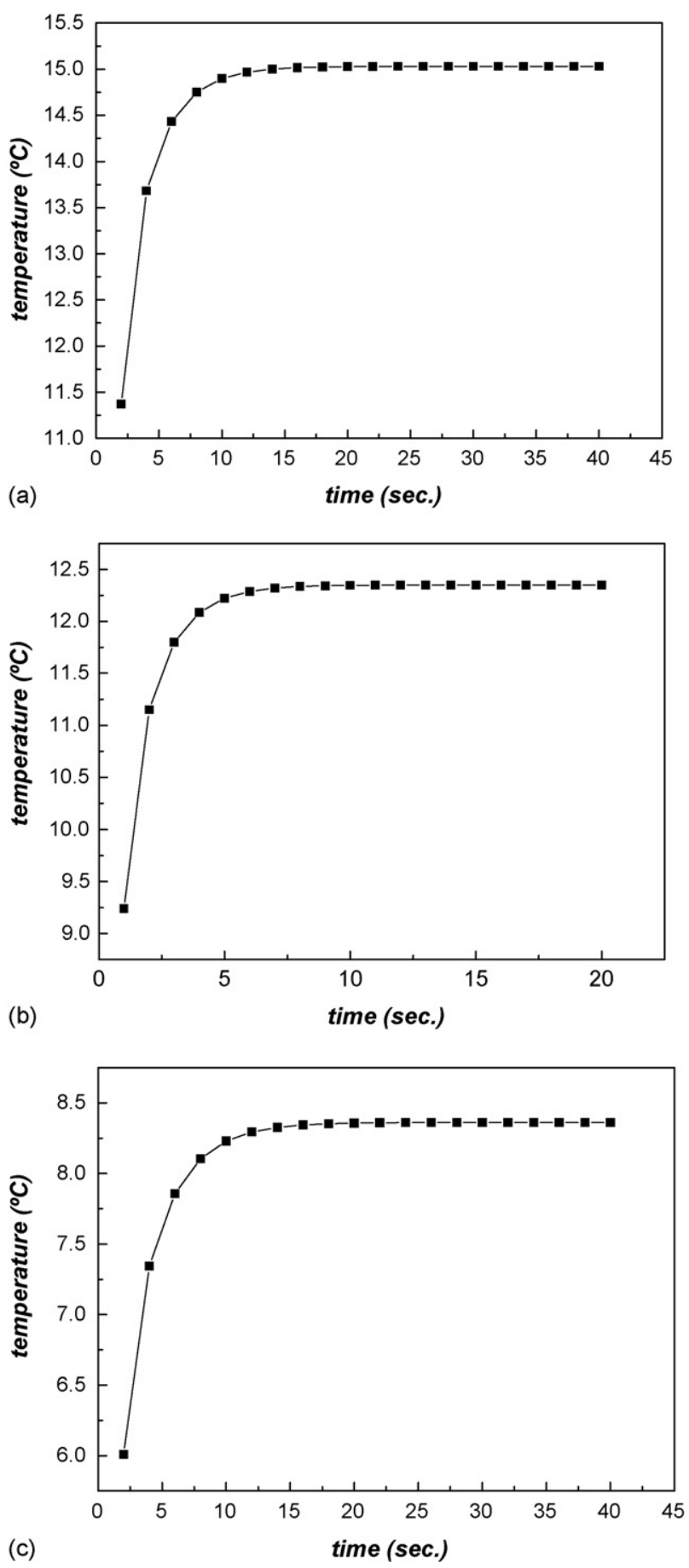

Fig. 9. ANSYS simulation results of temperature versus time of temperature sensor for different tested liquids (a) toluene, (b) ethanol and (c) glycerin.

transferring in the vertical direction is greater than that in the lateral direction due to the shape of the heater designed in this study. The higher the thermal diffusivity of the tested liquid, the more apparent is this phenomenon. Consequently, a smaller proportion of the heat flux transferring to the lateral sensor results in a larger time constant of the testing system. Table 2 shows the comparison between thermal diffusivity and time constant among the tested liquids. Table 3 shows the temperature change of experimental and simulation results. The temperature means
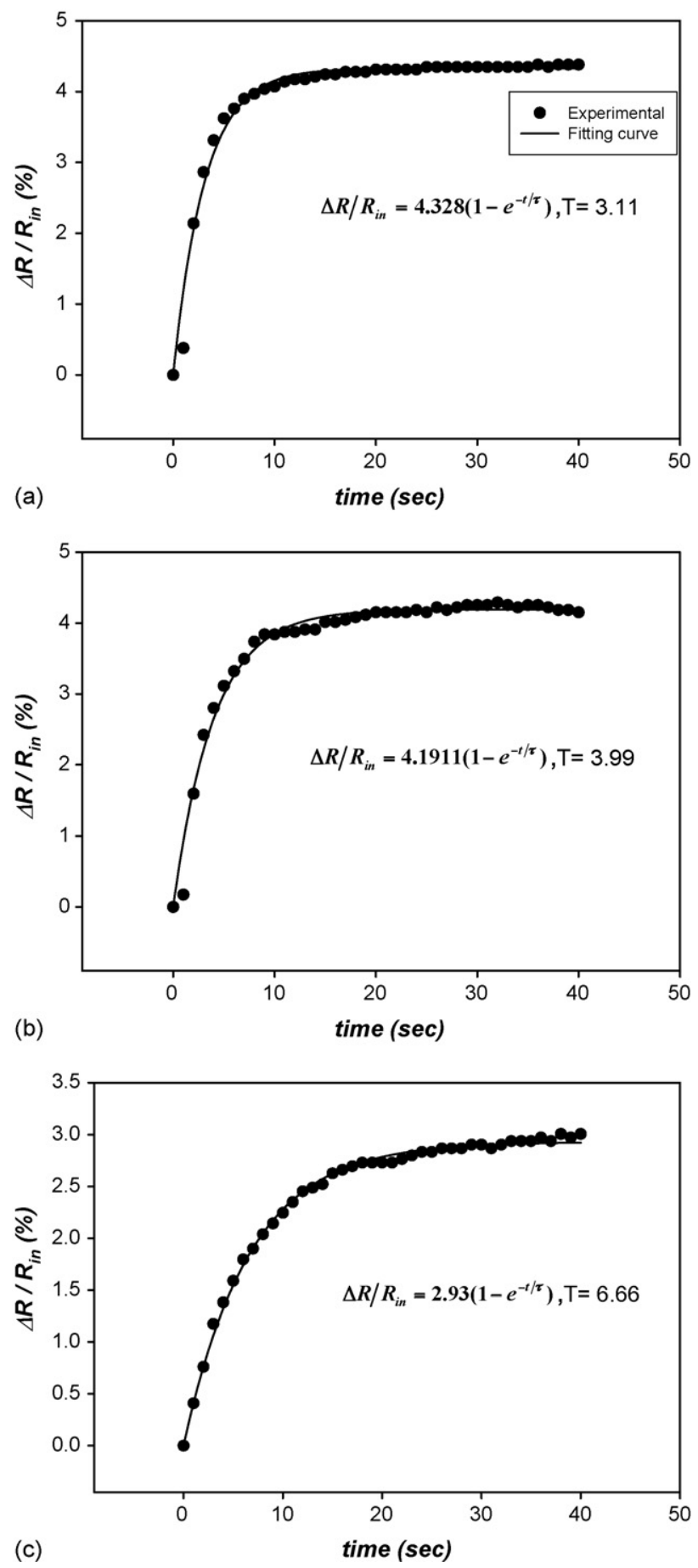

Fig. 10. Temporal response of resistance of temperature sensor versus time for measuring different tested liquids (a) toluene, (b) ethanol and (c) glycerin.

Table 2

The comparison of the thermal diffusivity and time constant among three tested liquids

\begin{tabular}{lcc}
\hline & $\alpha \times 10^{8}\left(\mathrm{~m}^{2} / \mathrm{s}\right)$ & $\tau(\mathrm{s})$ \\
\hline Toluene & 8.661 & 3.11 \\
Ethanol & 9.083 & 3.99 \\
Glycerin & 10.088 & 6.66 \\
\hline
\end{tabular}


Table 3

Temperature change of experimental and simulation results with an applied power of $0.01 \mathrm{~W}$ to the heater

\begin{tabular}{lccc}
\hline & $\alpha \times 10^{8}\left(\mathrm{~m}^{2} / \mathrm{s}\right)$ & $\Delta T_{\exp }(\mathrm{K})$ & $\Delta T_{\text {sim }}(\mathrm{K})$ \\
\hline Toluene & 8.661 & 11.51 & 15.03 \\
Ethanol & 9.083 & 10.91 & 12.35 \\
Glycerin & 10.088 & 7.89 & 8.36 \\
\hline
\end{tabular}

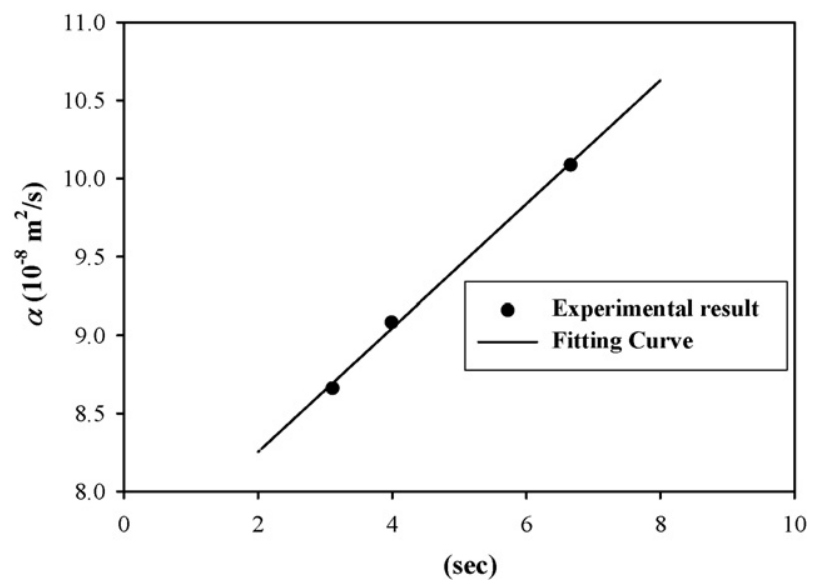

Fig. 11. The relation between thermal diffusivity and time constant of tested liquids.

the temperature difference between the steady temperature after the heat input and the initial temperature before the heat input. The temperature changes of experimental and ANSYS simulation results increase when thermal diffusivity of the tested liquids decreases. The temperature changes of simulation results are higher than that of experimental results because some heat loss in the experiments can not completely accounted for the simulation. The relation between thermal diffusivity and time constant among these liquids is illustrated in Fig. 11.

So far, a relation between thermal diffusivity and time constant among tested liquids is established by the measuring procedure in this study. For other liquids, their time constants could be measured by using the same procedure to obtain their thermal diffusivities.

\section{Conclusions}

A novel CMOS sensor chip for measuring thermal diffusivity of liquids is successfully designed, fabricated and tested. The present CMOS sensor chip is designed to solve three major challenges of measuring thermal diffusivity of liquids. Firstly, the present CMOS sensor chip, which is realized by VIS $0.5 \mu \mathrm{m}$ 2P3M CMOS process combined with post-CMOS micromachining processes, has higher yield rate than MEMS process. Secondly, the tested volume required for each liquid is only $3 \mu \mathrm{L}$. Thirdly, rapid diagnosis can be achieved since the entire measurement is performed on a single chip. This novel chip can identify thermal diffusivities of different tested liquids by observing the resistance variation of the temperature sensor, or the time constant of the experiment system. The experimental results of the measurement can be concluded as follows: as the heat flux generated from the heater is fixed, a liquid with higher thermal diffusivity will result in a smaller resistance variation of the temperature sensor. The time response of the experimental results can be characterized by a time constant. The experiment system will have a larger time constant when a liquid with higher thermal diffusivity is tested. Moreover, the thermal diffusivities of other liquids can be obtained through the measurement of their time constants based on the relation between thermal diffusivity and time constant established by the measuring procedure in this study. Reasonable agreement is also found between measured and simulated temperature responses of temperature sensor after the heat is applied to the heater.

\section{Acknowledgements}

The authors deeply appreciate Vanguard International Semiconductor Corporation (VIS) for the fabrication, and the NSC MEMS Research Center, Taipei, Taiwan for providing the equipment for post-micromachining. The work in this study could not have been achieved without their support.

\section{References}

[1] Y.S. Touloukian, P.E. Liley, S.C. Sexena, Thermal conductivity of nonmetallic liquids and gases, in: Thermal Properties of Matter, vol. 3, IFI/Plenum, New York/Washington, 1970.

[2] Y. Nagasaka, Y. Nagashima, Simultaneous measurement of the thermal conductivity and the thermal diffusity of liquids by the transient hot-wire method, Rev. Sci. Instrum. 52 (1981) 229-232.

[3] T. Tomimura, S. Maki, X. Zhang, M. Fuji, Measurements of thermal conductivity and thermal diffusivity of alternative refrigerants in liquid phase with a transient short-hot-wire method, Heat Transfer Asian Res. 33 (2004) $540-552$.

[4] Y. Nagasaka, Y. Nagashima, Absolute measurement of the thermal conductivity of electrically conducting liquids by the transient hot-wire method, J. Phys. E: Sci. Instrum. 14 (1981) 1435-1440.

[5] S.E. Gustafsson, E. Karawacki, M.N. Khan, Transient hot-strip method for simultaneously measuring thermal conductivity and thermal diffusivity of solids and fluids, J. Phys. D: Appl. Phys. 12 (1979) 1411-1421.

[6] H. Baltes, O. Paul, O. Brand, Micromachined thermally based CMOS microsensor, Proc. IEEE 86 (1998) 1660-1679.

[7] D. Jaeggi, H. Baltes, Thermoelectric AC power sensor by CMOS technology, IEEE Electron. Device Lett. 13 (1992) 366-368.

[8] T. Akin, Z. Olgun, O. Akar, H. Kulah, An integrated thermopile structure with high responsivity using any standard CMOS process, Sens. Actuators: Phys. 66 (1998) 218-224.

[9] J.-S. Kuo, W.S. Sun, P.H. Chen, Theoretical Analysis of a Novel Device for Measuring the Thermal Conductivity of Liquids, Int. Comm. Heat Mass Transfer 33 (2006) 426-433.

[10] P.H. Chen, H.Y. Peng, J.M. Hsieh, M.K. Chyu, The characteristic behavior of TMAH water solution for anisotropic etching on both silicon substrate and $\mathrm{SiO}_{2}$ layer, Sens. Actuators: Phys. 93 (2001) 132-137.

[11] Y.T. Cheng, C.C. Pun, C.Y. Tsai, P.H. Chen, An array-based CMOS biochip for electrical detection of DNA with multilayer self-assembly gold nanoparticles, Sens. Actuators: Chem. 109 (2005) 249-255.

[12] Y.T. Cheng, C.Y. Tsai, P.H. Chen, Development of an Integrated CMOS DNA Detection Biochip, Sens. Actuators: Chem., in press. 